\title{
Current status and future direction of community-based management of hypertension in China
}

\author{
Yingxian Sun, Guozhe Sun, Ye Chang \\ Department of Cardiology, The First Hospital of China Medical University, Shenyang 110001, Liaoning Province, \\ China
}

Hypertension is one of the most prevalent chronic diseases, with about 244.5 million patients in China currently. ${ }^{[1]}$ It is the key risk factor for cardiovascular and cerebrovascular deaths and seriously affects people's health and economic and social development. ${ }^{[2]}$ It is of great importance to improve the control rate of hypertension and decrease its complications.

\section{INCREASING} HYPERTENSION PREVALENCE AND BURDEN OF ITS COMPLICATION

Since the 1950s in China, six large-scale hypertension surveys have been conducted to explore its epidemiological characteristics, and it was found that the prevalence of hypertension raised from $5.1 \%$ to $23.2 \% .^{[1,3]}$ It is worth noting that the prevalence of hypertension in rural areas has already exceeded that in urban areas and the rural-urban gap is still expanding. ${ }^{[4]}$ Also hypertension is more often seen than before among young individuals, with prevalence rates of $3.5 \%, 5.8 \%$, and $14.1 \%$ in people aged 18-24, 25-34, and 35-44 years of age, respectively. ${ }^{[5]}$ Our previous study showed that the incidence of hypertension in a rural area had reached as high as $11.4 \%$ in general population. ${ }^{[6]}$ It is predictable that the prevalence of hypertension will continue to increase in the future in that and other similar areas. Disappointedly, with the high prevalence, the current awareness, treatment, and control rates are just $51.6 \%$, $45.8 \%$, and $16.8 \%$, respectively, which are better than those in 2002 (30.2\%, 24.7\%, and $6.1 \%$, respectively), ${ }^{[3]}$ but they are still at a very low level. Hypertension detection and medication use in rural adults are far from optimal. Therefore, we should pay attention to hypertension people in all the regions and all the age groups.

According to a research reported by Chinese Center for Disease Control and Prevention, the number of deaths from hypertension in China reached 2.54 million in 2017. About $69 \%$ of deaths from stroke, $54 \%$ of deaths from ischemic heart diseases, and $43 \%$ of deaths from chronic kidney diseases could be attributed to hypertension. ${ }^{[2]}$ Therefore, it is a crucial to control hypertension in China. ${ }^{[1,7]}$

\section{DEVELOPMENT OF COMMUNITY-BASED HYPERTENSION CONTROL}

Elevated blood pressure is the most commonly seen chronic condition in the practicing physician's office. What the practicing physician does not generally realize, however, is that the portion of the hypertensive patients that he sees in his office is only the "tip of the iceberg" and that the bulk of the patients is not detected except when community surveys are made. ${ }^{[8]}$ The United States National Health Survey in 1960-1962 indicated that the awareness, treatment, and control rates of hypertension were only $15.2 \%, 23.2 \%$, $16.3 \%$, respectively, ${ }^{[8]}$ which was comparable to the results of Framingham study and other previous studies. Community hypertensive surveys have shown that the majority of people with elevated blood pressure are either undetected, untreated, or inadequately treated. As early as in 1969, 
Wilber reported his community-based intervention of hypertension research and found that intervention program could improve hypertension control rates. ${ }^{[9]}$ Since then, Wilber and other researchers conducted more studies and supported this finding. In 1972, he published an article titled "Hypertension - A Community Problem," indicating the importance of community-based hypertension prevention. ${ }^{[8]}$ It switched from traditional outpatient model of patient-doctor relationship to communitybased hypertension management, which can achieve better control of hypertension. Therefore, the 1984 Report of the Joint National Committee on Detection, Evaluation, and Treatment of High Blood Pressure first proposed community programs of hypertension treatment in all guidelines. This guideline indicated that patient adherence and the maintenance of a therapeutic regimen was a major challenge. Furthermore, community-based hypertension control programs, with many groups constituting them, continue to offer referral, follow-up, and monitoring services, complementing medical management by private physicians. ${ }^{[10]}$ Community-based programs could detect more hypertension patients who were unaware of their condition, and greatly improved the overall hypertension control rate. In 2017, the ACC/AHA Guideline for the Prevention, Detection, Evaluation, and Management of High Blood Pressure in Adults stated that integration of social and community services offered complementary reinforcement of clinically identified treatment goals. Social and community services are helpful when explicitly related to medical care. ${ }^{[1]}$ All these strategies can improve hypertension control rate.

In China, the first social community-based hypertension control study was conducted in 1969 by Professor Yingkai Wu and Lisheng Liu in Shougang workplace and community. They found that social community screening combined with simplified diagnostic evaluation, patient education, and follow-up visits could remarkably increase the percentages of treatment and control. ${ }^{[12]}$ Since then, some community-based research studies were made for the prevention and treatment of hypertension. With the accumulating evidence, the 2010 version of Chinese guideline of hypertension management highlighted the community management strategy of hypertension and recommended that community-based standardized management of hypertension was the fundamental way to improve the awareness, treatment, and control rates. ${ }^{[13]}$ It highly recommends the community-based management of hypertension among all the guidelines.

\section{IMPLEMENTATION STUDIES ON COMMUNITY HYPERTENSION CONTROL FILL IN THE GAP}

\section{BETWEEN KNOWLEDGE AND HYPERTENSION CONTROL RESULTS}

Based on the accumulated studies, it is known that hypertensive patients can benefit from blood pressure reduction and that community-based strategy is essential to reduce blood pressure. However, hypertension control rates are still very low worldwide (only $13.8 \%$ in global), ${ }^{[14]}$ especially in low- and middle-income groups. There is an urgent need to know how to improve hypertension control rate through community-based hypertension management. From the beginning of this century, a series of implementation studies on community-based hypertension control and management were conducted worldwide and remarkable achievements have been made. In America, a study in black barbershops indicated that health promotion by barbers resulted in sharp blood pressure reduction when coupled with medication management in barbershops by specialty-trained pharmacists. A blood pressure level of less than $130 / 80 \mathrm{mmHg}$ was achieved among $63.6 \%$ of the participants in the intervention group. ${ }^{[15]}$ People of lowincome group in Argentina with uncontrolled hypertension who participated in a multicomponent intervention experienced a greater decrease in both systolic and diastolic blood pressure than patients who received usual care over 18 months. The proportion of patients with controlled hypertension increased from $17.0 \%$ at baseline to $72.9 \%$ at 18 months in the intervention group. ${ }^{[16]}$ In rural communities in Bangladesh, Pakistan, and Sri Lanka, a multicomponent intervention that was centered on proactive home visits by trained government community health workers who were linked with existing public health care infrastructure led to a greater reduction in blood pressure than usual care among adults with hypertension. Blood pressure control $(<140 / 90$ $\mathrm{mmHg}$ ) was achieved in $53.2 \%$ of the participants in the intervention group. ${ }^{[17]}$

Compared with these and some other countries, China has its own characteristics. There are community hospitals in the cities, and township hospitals and village doctors in the rural areas. Many studies have been carried out in urban communities and functional communities in China. Recently, a study conducted by Wang et al. confirmed that workplace-based multicomponent intervention on hypertension control appeared to be more effective than usual care, leading to measurable benefits such as lower blood pressure, improved hypertension control, and adoption of healthy lifestyle habits. The hypertension control rate in the intervention group was $66.2 \% .^{[18]}$ The study in rural community with good control is rare. Our team has been engaged in the prevention and treatment of hypertension in Chinese rural areas since 2003 and carried out cluster randomized trials in rural China. We found a 
serial model for hypertension treatment and management, which was suitable for rural China on the basis of low-cost anti-hypertensive drugs. ${ }^{[1,20]}$ All those efforts are improving hypertension control in China.

\section{FUTURE DIRECTION OF COMMUNITY-BASED MANAGEMENT OF HYPERTENSION IN CHINA}

In some cities such as Shanghai and Hangzhou in China, hypertension control rates reach up to $50 \%$ or even to $70 \%$, which is close to that in the developed countries. However, for most of the other cities, especially the rural areas, the hypertension control rate remained extremely low. We need more implementation studies and apply the results and benefits in more communities in the cities and rural areas to improve the hypertension control rate. In order to improve the overall level of hypertension control in China, we need to carry out multifaceted interventions in these areas at health system level, doctor level, and patient level. Promisingly, China has introduced many favorable policies recently and the existing problems are being solved step by step.

The government has introduced many policies on the management of hypertension. First, a goal has been set up in the framework of the Healthy China 2030 Plan, which is a programmatic document in the field of health and is a great support in hypertension management. By 2030 , the awareness rate of hypertension among residents aged 30 years and above should not be lower than $65 \%$; the management rate should not be lower than $70 \%$; the treatment rate and control rate of hypertension should continue to be improved; and the cardiovascular and cerebrovascular mortality rate should drop from 238.4 to 190.7 per $100,000 .{ }^{[21]}$ It is challenging but possible. Also, the General Office of the State Council launched a file about hierarchical diagnosis and treatment system, which required that the first visit and routine management of hypertension must be in the community hospital or clinic in the villages. Furthermore, decreasing price of the antihypertensive medicine by government enables most of the patients to afford the cost. The Healthy China 2030 Plan coupled with the implementation studies will greatly improve the hypertension control rate. With these policy-level supports to provide guidance and implementation studies to provide strategic and technical evidence, we will accumulate more experience in the management of hypertension, improve the control rate of hypertension, and achieve the goals of the Healtby Cbina 2030 Plan.

\section{Conflict of Interest}

None declared.

\section{REFERENCES}

1. Wang Z, Chen Z, Zhang L, Wang X, Hao G, Zhang Z, et al. Status of Hypertension in China: Results From the China Hypertension Survey, 2012-2015. Circulation 2018; 137: 2344-56.

2. Zhou M, Wang H, Zeng X, Yin P, Zhu J, Chen W, et al. Mortality, morbidity, and risk factors in China and its provinces, 1990-2017: a systematic analysis for the Global Burden of Disease Study 2017. Lancet (London, England) 2019, 394: 1145-58.

3. 2018 Chinese Guidelines for Prevention and Treatment of Hypertension-A report of the Revision Committee of Chinese Guidelines for Prevention and Treatment of Hypertension. J Geriatr Cardiol 2019, 16: 182-241.

4. Li J, Shi L, Li S, Xu L, Qin W, Wang H. Urban-rural disparities in hypertension prevalence, detection, and medication use among Chinese Adults from 1993 to 2011. Int J Equity Health 2017, 16: 50.

5. Guidelines for the Prevention and Treatment of Hypertension in China (2018 Revision). Chin J Cardiol 2019, 24: 24-56.

6. Sun Z, Zheng L, Detrano R, Zhang X, Xu C, Li J, et al. Incidence and predictors of hypertension among rural Chinese adults: results from Liaoning province. Ann Fam Med 2010, 8: 19-24.

7. Su M, Zhang Q, Bai X, Wu C, Li Y, Mossialos E, et al. Availability, cost, and prescription patterns of antihypertensive medications in primary health care in China: a nationwide cross-sectional survey. Lancet (London, England) 2017, 390: 2559-68

8. Wilber JA, Barrow JG. Hypertension--a community problem. Am J Med 1972, 52: 653-63.

9. Wilber JA, Barrow JG. Reducing elevated blood pressure. Experience found in a community. Minn Med 1969, 52: 1303-6.

10. The 1984 Report of the Joint National Committee on Detection, Evaluation, and Treatment of High Blood Pressure. Arch Int Med 1984, 144: 1045-57.

11. Whelton PK, Carey RM, Aronow WS, Casey DE, Collins KJ, Dennison Himmelfarb C, et al. 2017 ACC/AHA/AAPA/ABC/ACPM/AGS/APhA/ ASH/ASPC/NMA/PCNA Guideline for the Prevention, Detection, Evaluation, and Management of High Blood Pressure in Adults: A Report of the American College of Cardiology/American Heart Association Task Force on Clinical Practice Guidelines. Hypertension 2018: 71: e13-e115.

12. Wu XG, Gu DF, Wu YF, Yu XH, Wang SY, Wang N, et al. [An evaluation on effectiveness of worksite-based intervention for cardiovascular disease during 1974 - 1998 in capital iron and steel company of Beijing]. Zhonghua Yu Fang Yi Xue Za Zhi 2003, 37: 93-7.

13. Liu LS. [2010 Chinese guidelines for the management of hypertension]. Zhonghua Xin Xue Guan Bing Za Zhi 2011, 39: 579-615.

14. Mills KT, Stefanescu A, He J. The global epidemiology of hypertension. Nat Rev Nephrol 2020, 16: 223-37.

15. Victor RG, Lynch K, Li N, Blyler C, Muhammad E, Handler J, et al. A Cluster-Randomized Trial of Blood-Pressure Reduction in Black Barbershops. N Engl J Med 2018, 378: 1291-301.

16. He J, Irazola V, Mills KT, Poggio R, Beratarrechea A, Dolan J, et al. Effect of a Community Health Worker-Led Multicomponent Intervention on Blood Pressure Control in Low-Income Patients in Argentina: A Randomized Clinical Trial. JAMA 2017, 318: 1016-25.

17. Jafar TH, Gandhi M, de Silva HA, Jehan I, Naheed A, Finkelstein EA, et al. A Community-Based Intervention for Managing Hypertension in Rural South Asia. N Engl J Med 2020, 382: 717-26.

18. Wang Z, Wang X, Shen Y, Li S, Chen Z, Zheng C, et al. Effect of a Workplace-Based Multicomponent Intervention on Hypertension Control: A Randomized Clinical Trial. JAMA Cardiol 2020, 5: 567-75.

19. Yu S, Guo X, Yang H, Zheng L, Sun Y. Cardiometabolic comorbidities and epidemiological features among rural Chinese elderly people. Aging Clin Exp Res 2020, 32: 1777-88. 
20. Zheng J, Sun ZQ, Guo XF, Xie YX, Sun YX, Zheng LQ. Blood pressure predictors of stroke in rural Chinese dwellers with hypertension: a largescale prospective cohort study. BMC Cardiovasc Disord 2019, 19: 206.
21. CPC Central Committee, State Council. The plan for "Healthy China 2030". 2019. Available at: http://www.gov.cn/xinwen/2019-07/15/ content_5409694.htm (accessed Jul 15, 2019).

How to cite this article: Sun $Y$, Sun G, Chang Y. Current status and future direction of community-based management of hypertension in China. J TransI Intern Med 2021; 9: 61-4. 